\title{
RUTAS DE TRANSPORTE PÚBLICO Y SITUACIÓN DE LA TUBERCULOSIS EN LIMA, PERÚ
}

\author{
Octavio Garaycochea1,a, Eduardo Ticona $2,3, \mathrm{a}, \mathrm{b}$
}

\begin{abstract}
RESUMEN
Con el objetivo de determinar la asociación entre el número de rutas formales de transporte público urbano y la situación de la tuberculosis (TB) en cada distrito de Lima, se realizó un análisis correlacional y un modelo de regresión lineal entre ambas variables. Asimismo, se determinó el número de rutas de transporte público de mayor probabilidad de transmisión de TB, las zonas de mayor circulación de dichas rutas y un mapeo de estas. Existe una correlación positiva entre el número de rutas formales que recorren cada distrito de Lima y la situación de TB; un porcentaje de la situación de TB podría ser explicada por el número de rutas de transporte público. De las 404 rutas formales que recorren Lima Metropolitana, $147(36,4 \%)$ son rutas de mayor probabilidad de transmisión de TB. El transporte público debería ser considerado en la lucha actual contra la TB.
\end{abstract}

Palabras clave: Tuberculosis; transportes; Vigilancia Sanitaria; Perú (fuente: DeCS BIREME)

\section{ROUTES OF PUBLIC TRANSPORT AND THE SITUATION OF TUBERCULOSIS IN LIMA, PERU}

\begin{abstract}
In order to determine the association between the number of formal urban public transport routes and the situation of tuberculosis (TB) in each district of Lima, a correlational analysis and linear regression model between the two variables was performed. Also determined were the number of public transport routes most likely to transmit TB, the areas of greatest activity of these routes and a mapping of these routes. There is a positive correlation between the number of formal routes through each district of Lima and the situation of tuberculosis; a percentage of the TB situation could be explained by the number of public transport routes. Of the 404 formal routes that run through Lima, $147(36.4 \%)$ are routes of greater probability of transmission of TB. Public transport should be considered in the ongoing fight against TB.
\end{abstract}

Key words: Tuberculosis, transportation, Health Surveillance, Peru (source: MeSH, NLM)

\section{INTRODUCCIÓN}

El año 2012 se reportaron en el mundo 8,6 millones de casos nuevos de tuberculosis (TB) ${ }^{(1)}$, en la región de las Américas el $60 \%$ de todos los casos nuevos se registraron en Brasil, Perú, México y Haití (2). El Perú, es el segundo país con la carga de TB más alta de la región con 32405 casos reportados el $2011^{(3)}$. Sin embargo, la magnitud de la endemia de TB en el Perú no es uniforme ya que el $55,8 \%$ de casos de TB, el $80 \%$ de casos TB MDR y el $89 \%$ de casos de TB XDR son reportados en Lima y Callao ${ }^{(3)}$. Específicamente, en Lima el $86 \%$ de los casos de TB se notifican en 18 de sus 43 distritos ${ }^{(4)}$.
Un tercio de la población nacional vive en Lima, una ciudad densamente poblada con $2300 \mathrm{hab} / \mathrm{km}^{2}$ (Perú $\left.18 \mathrm{hab} / \mathrm{km}^{2}\right)^{(5)}$. El transporte público en Lima está conformado por una línea del tren eléctrico, una ruta troncal del sistema de buses metropolitano y el resto lo conforman las compañías de vehículos de transporte público urbano. Estas últimas, no cuentan con un tipo de vehículo estándar, pues emplean tres tipos de vehículos: camionetas rurales ("combis"), microbuses y ómnibus. Lima concentra el $68,5 \%$ del parque automotor nacional lo cual genera alrededor de 10 millones de viajes al día de los cuales el $80 \%$, se realizan en transporte público ${ }^{(5)}$.

\footnotetext{
Universidad de San Martín de Porres. Lima, Perú.

Hospital Nacional Dos de Mayo. Lima, Perú.

Universidad Nacional Mayor de San Marcos. Lima, Perú.

Médico cirujano ${ }^{\mathrm{b}}$ máster en Ciencias Tropicales Biomédicas

Recibido: 16-08-14 Aprobado: 19-11-14
}

Citar como: Garaycochea O, Ticona E. Rutas de transporte público y situación de la tuberculosis en Lima, Perú. Rev Peru Med Exp Salud Publica. 2015;32(1):937. 
Al ser la TB una enfermedad infectocontagiosa que se transmite por vía respiratoria ${ }^{(1)}$, y cuyo riesgo de infección aumenta en lugares hacinados como los vehículos de transporte público ${ }^{(6)}$, el presente estudio buscó determinar la asociación entre el número de rutas formales de transporte público urbano y la situación de la TB en cada distrito de Lima. Asimismo, se buscó identificar las rutas de transporte público urbano de mayor probabilidad de transmisión de TB y las zonas de mayor circulación de estas.

\section{EL ESTUDIO}

El estudio realizado fue de tipo ecológico, se obtuvo información de la GTU (Gerencia de Transporte Urbano de la Municipalidad de Lima Metropolitana) sobre las compañías de vehículos de transporte público urbano del 2011: número de rutas de transporte público, itinerario y tipo de vehículo empleado por estas (ómnibus, microbús y "combi" o camioneta rural). Una ruta de transporte público se definió como un trayecto determinado que recorre un vehículo prestador de servicio de transporte a través de la ciudad.

El perfil epidemiológico de la TB en Lima - 2011 fue obtenido de la Estrategia Sanitaria Nacional de Prevención y Control de la Tuberculosis (ESNPCT) del Ministerio de Salud (MINSA). La situación de TB fue descrita por cuatro variables: total de casos de TB en todas sus formas (TB no pulmonar y TB pulmonar frotis negativo); tasa de incidencia (por 100000 habitantes); tasa de incidencia TB pulmonar frotis positivo BK+ (por 100000 habitantes), y tasa de morbilidad (por 100000 habitantes) (Tabla 1). La definición de tasa de morbilidad empleada por la ESNPCT-MINSA es una definición local que equivale a la prevalencia de TB.
Los distritos con mayores índices de TB se determinaron a partir del Reporte de la Situación de Tuberculosis en el Perú ESNPCT (2008) (4), dicho reporte fue el último disponible que definió a los distritos de Lima más endémicos, los cuales, adicionalmente a los indicadores mencionados, consideran el número de casos TB-MDR y porcentaje de hacinamiento.

Se definió como rutas de mayor probabilidad de transmisión de TB (rutas-MPTB) a aquellas rutas que realizaban íntegramente todo su recorrido en uno o más de los 18 distritos con mayores índices de TB, y si pasaban por distritos con bajo índice de TB, solo lo hacían a través de vías de alta velocidad (Vía de Evitamiento y Carretera Panamericana).

Con la información obtenida se determinó el número de rutas por distrito, el número de rutas de mayor probabilidad de transmisión de TB y el tipo de vehículo más empleado por ambas. Una vez definidas las rutas de mayor probabilidad de transmisión de TB se determinó las zonas de mayor circulación de las mismas y se realizó un mapeo de estas utilizando el software de sistema de Información geográfica ArcGIS.

Se realizó una correlación de Pearson o Spearman según la distribución de las variables entre el número de rutas formales de transporte público y la situación de la tuberculosis en cada distrito para determinar el grado de asociación y dirección entre ambas. De la misma forma, se realizó una correlación entre las rutas-MPTB y los respectivos indicadores de la situación de TB, para determinar si es que existía una mayor asociación en comparación al total de rutas. Con el fin de determinar si la situación de la TB podría deberse en parte al número

Tabla 1. Distritos de Lima con mayores índices de tuberculosis, 2011

\begin{tabular}{|c|c|c|c|c|}
\hline Distritos & $\begin{array}{l}\text { Casos de tuberculosis en } \\
\text { todas sus formas }\end{array}$ & Tasa de incidencia & $\begin{array}{c}\text { Tasa de } \\
\text { incidencia BK+ }\end{array}$ & $\begin{array}{l}\text { Tasa de } \\
\text { morbilidad }\end{array}$ \\
\hline San Juan de Lurigancho & 2095 & 190,6 & 128,7 & 208,59 \\
\hline Ate & 1094 & 175,5 & 93,5 & 196,77 \\
\hline San Martín de Porres & 857 & 118,9 & 64,7 & 132,62 \\
\hline Comas & 826 & 141,7 & 71,2 & 160,31 \\
\hline Villa María del Triunfo & 652 & 155,58 & 98,55 & 155,58 \\
\hline El Agustino & 599 & 276,8 & 164,3 & 316,38 \\
\hline La Victoria & 593 & 263,2 & 163,8 & 318,53 \\
\hline Villa el Salvador & 587 & 137,32 & 85,39 & 137,32 \\
\hline San Juan de Miraflores & 579 & 148,53 & 95,94 & 148,53 \\
\hline Lima & 517 & 147,3 & 90,5 & 177,15 \\
\hline Santa Anita & 476 & 205,6 & 123,6 & 228,08 \\
\hline Rímac & 383 & 219,84 & 192,3 & 111,9 \\
\hline
\end{tabular}




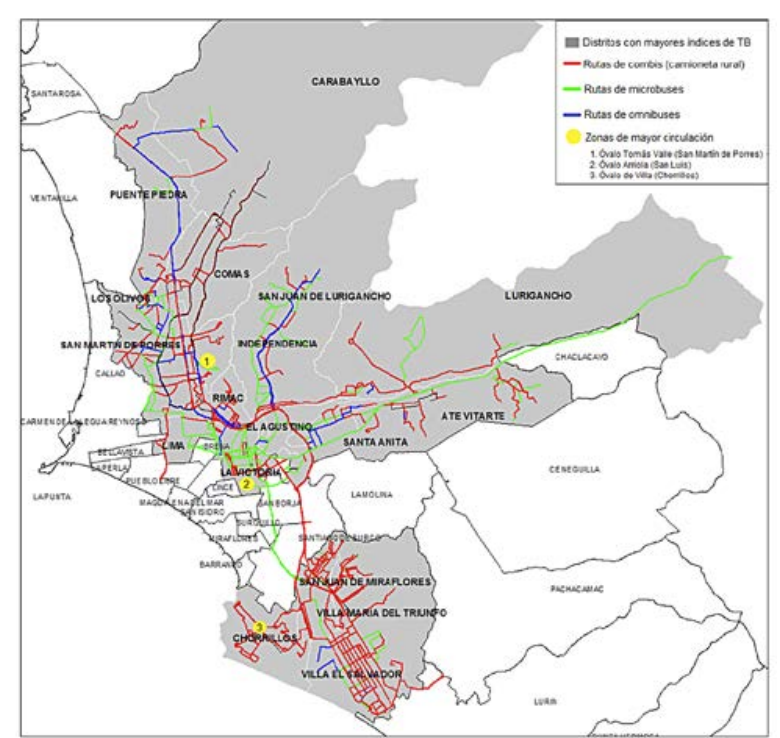

Figura 1. Rutas de transporte público de mayor probabilidad de transmisión de tuberculosis

de rutas formales de transporte público se realizó un análisis de regresión lineal entre ambas variables. Los análisis estadísticos se llevaron a cabo utilizando el paquete estadístico SPSS versión 20 para Windows, con un nivel de confianza de $95 \%$ (valor $p<0,05$ se consideró significativo).

\section{HALLAZGOS}

Lima Cercado es el distrito por el cual discurren la mayor cantidad de rutas de transporte público (236), seguido de La Victoria (179), San Martín de Porres (165) y Rímac (159). La media de las rutas por distrito es 68,8 y la desviación estándar es de 56,05.

De las 404 rutas que recorren Lima Metropolitana, 147 $(36,4 \%)$ fueron determinadas como rutas-MPTB. Las zonas de mayor circulación de estas fueron: Óvalo Arriola (San Luis) 17\%; Óvalo de Villa (Chorrillos) $5 \%$, y óvalo Tomas Valle (San Martín de Porres) $2 \%$ (Figura 1).

Respecto al tipo de vehículo de las rutas-MPTB; 99 $(67,3 \%)$ emplean "combis"; 33 (22,4\%) microbuses y 15 $(10,2 \%)$ ómnibus. Sin embargo, la distribución del tipo de vehículo del total de rutas es: $168(41,6 \%)$ emplean "combis", 167 (41,3\%) microbuses y $69(17,1 \%)$ ómnibus.

Se obtuvo una correlación positiva entre el número de rutas de cada distrito y cada uno de los indicadores de la situación de TB empleados. De la misma forma, se
Tabla 2. Tabla de correlaciones

\begin{tabular}{|c|c|c|}
\hline Variables correlacionadas & Correlación & Valor $p$ \\
\hline $\begin{array}{l}\text { Rutas por distrito - total de casos } \\
\text { TB en todas sus formas }\end{array}$ & 0,518 & $<0,001^{*}$ \\
\hline $\begin{array}{l}\text { Rutas por distrito - tasa de } \\
\text { incidencia }\end{array}$ & 0,401 & $0,008^{* *}$ \\
\hline $\begin{array}{l}\text { Rutas por distrito - tasa de inci- } \\
\text { dencia BK+ }\end{array}$ & 0,398 & $0,008^{* *}$ \\
\hline $\begin{array}{l}\text { Rutas por distrito - tasa de } \\
\text { morbilidad }\end{array}$ & 0,406 & $0,007^{\star *}$ \\
\hline $\begin{array}{l}\text { Rutas TB - total de casos TB en } \\
\text { todas sus forma }\end{array}$ & 0,662 & $<0,001^{*}$ \\
\hline Rutas TB - tasa de incidencia & 0,613 & $<0,001^{* *}$ \\
\hline $\begin{array}{l}\text { Rutas TB - tasa de incidencia } \\
\text { BK+ }\end{array}$ & 0,59 & $<0,001^{* *}$ \\
\hline Rutas TB - tasa de morbilidad & 0,6 & $<0,001^{* *}$ \\
\hline
\end{tabular}

obtuvo una correlación positiva y con valores más altos entre el total de rutas-MPTB por distrito y la situación de tuberculosis de cada uno (Tabla 2).

En el análisis de regresión lineal se observó en todos los casos una pendiente positiva con un coeficiente de determinación también positivo: $r^{2}=0,268$ total de casos de TB en todas su formas/rutas por distrito $\left(r^{2}\right.$ corregida $0,250) ; r^{2}=0,167$ tasa de incidencia/rutas por distrito $\left(r^{2}\right.$ corregida 0,147$) ; r^{2}=0,174$ tasa de incidencia $B K+1$ rutas por distrito ( $r^{2}$ corregida 0,154$) ; r^{2}=0,196$ tasa de morbilidad/rutas por distrito $\left(r^{2}\right.$ corregida 0,176$)$.

\section{DISCUSIÓN}

El estudio evidencia una correlación directa y, por lo tanto, una asociación directa entre el número de rutas de transporte público y la situación de TB de cada distrito, lo que concuerda con reportes de estudios de búsqueda de casos luego del diagnóstico de un caso de TB activa (7), estudios matemáticos de predicción del riesgo de infección de TB en vehículos de transporte público ${ }^{(8)}$ así como estudios analíticos y estudios espaciales ${ }^{(6-9)}$. En el Perú Horna-Campos et al. han descrito también esta relación ${ }^{(10)}$ y el riesgo de contagio no solo en los usuarios, sino también en los mismos trabajadores del transporte público ${ }^{(11,12)}$.

Si bien los coeficientes de determinación obtenidos a partir de los análisis de regresión lineal fueron positivos, pero con valores bajos, esta positividad guarda relación con los resultados del análisis correlacional, y a partir de estos podríamos deducir que un porcentaje de la situación de TB en Lima podría ser explicado por el número de rutas de transporte público. Sin embargo, esta 
evidencia es relativa debido al tipo de estudio realizado y a los múltiples factores de riesgo relacionados a la infección por TB ${ }^{(9)}$

Los valores de la correlación entre la situación de TB de cada distrito y las rutas-MPTB fueron más altos que los obtenidos con el total de rutas. Este análisis más profundo demuestra que si bien ya se ha concluido que existe una correlación positiva entre las rutas de transporte público que recorren cada distrito de Lima y la situación de TB de este; dentro de cada distrito de Lima, y por ende en Lima Metropolitana, las rutas-MPTB están más relacionadas con la situación de tuberculosis que el total de rutas. En estas rutas que recorren los 18 distritos con peor situación de TB, posiblemente los trabajadores del transporte y los usuarios, tengan mayor riesgo de infección por TB.

Respecto al tiempo estimado de transmisión en vehículos de transporte público, en un estudio realizado en Perú (12) se evidenció que la infección tuberculosa fue mayor en los trabajadores que laboraban más de 60 horas por semana. Otro estudio demostró que en usuarios que viajaron más de 90 minutos en un vehículo en el que el conductor tenía un cuadro de TB activa, el $11 \%$ tuvo un PPD positivo ${ }^{(13)}$.

La camioneta rural o "combi" es el tipo de vehículo más empleado por el total de rutas, y en particular en las rutas-MPTB. Este vehículo es de dimensiones más pequeñas $y$, por lo tanto, de menor volumen cúbico ${ }^{(14)}$, por lo que existiría un mayor riesgo de infección entre sus ocupantes si una persona con TB activa se encuentra en el vehículo, inclusive sin sobresaturar su capacidad, la que generalmente es superada en las horas punta del tráfico y en particular en las mañanas, momento que las personas con TB poseen mayor expectoración ${ }^{(10-15)}$.
Las principales limitaciones del estudio fueron que al ser de tipo ecológico, está afecto a la falacia ecológica y a la dificultad de controlar factores de confusión; se determinó asociación y no causalidad entre ambas variables y solo se analizó la variable transporte público y no otros factores asociados a la infección de tuberculosis. No se incluyó en el análisis de correlación ni de regresión la rutas inscritas en la provincia institucional del Callao, las rutas informales (La GTU no cuenta con el dato exacto de que porcentaje representan estas del total de rutas y vehículos que circulan en Lima) el tiempo de permanencia de los pasajeros, los tiempos de recorrido de las rutas y el tipo de vehículo empleado.

El transporte público es un factor que se debe tener en cuenta en la lucha horizontal contra la TB. Es aceptado que Lima requiere de una reforma de transporte público por diferentes factores, a los que sería conveniente adicionar la variable de salud pública, relacionada con disminuir el riesgo de exposición de TB reduciendo los tipos de vehículos más empleados ("combis"); reordenando rutas, puesto que muchas de estas tienen recorridos similares; sin permitir llevar más pasajeros de lo aceptado, con prioridad en las rutas-MPTB. Así mismo, los vehículos de transporte público deberían ser usados como espacios para la prevención y promoción de salud. Los resultados obtenidos en este estudio ecológico pueden ser la base de futuros estudios de mayor complejidad.

Contribuciones de los autores: ambos autores han participado en la concepción y diseño del artículo, análisis e interpretación de datos, redacción del artículo, revisión crítica del artículo y asesoría estadística. Además, OG aportó el material de estudio y recolectó los resultados; ET aprobó la versión final.

Conflictos de interés: los autores declaran no tener conflictos de interés.

Fuentes de financiamiento: autofinanciado.

\section{REFERENCIAS BIBLIOGRÁFICAS}

1. World Health Organization. Global tuberculosis report 2012. Geneva: WHO; 2012.

2. Organización Panamericana de la Salud. La tuberculosis en la región de las Américas: Informe Regional 2012. Epidemiología, control y financiamiento. Washington D.C.: OPS; 2013.

3. Alarcón Guizado, Antonieta. Situación epidemiológica de la tuberculosis Perú. Al primer semestre del 2012. Lima: ESNPCT-MINSA; 2012.
4. Bonilla Asalde, Cesar. Situación de la tuberculosis en el Perú. Acta Med Per. 2008;25(3):163-170.

5. Gerencia de Transporte Urbano. Municipalidad Metropolitana de Lima [Internet]. Lima: GTU; 2013 [citado el 14 de noviembre de 2013]. Disponible en: http://www.gtu. munlima.gob.pe/

6. Feske ML, Teeter LD, Musser JM, Graviss EA. Giving TB wheels: Public transportation as a risk factor for tuberculosis transmission. Tuberculosis
(Edinb). 2011 Dec;91 Suppl 1:S16-23. doi: 10.1016/j.tube.2011.10.005.

7. Edelson PJ, Phypers M. TB transmission on public transportation: a review of published studies and recommendations for contact tracing. Travel Med Infect Dis. 2011 Jan;9(1):27-31. doi: 10.1016/j. tmaid.2010.11.001.

8. Castillo-Chavez C, Song B. Dynamical models of tuberculosis and their applications. Math Biosci Eng. 2004 Sep;1(2):361-404. 
9. Feske ML, Teeter LD, Musser JM, Graviss EA. Including the third dimension: a spatial analysis of $\mathrm{TB}$ cases in Houston Harris County. Tuberculosis (Edinb). 2011 Dec;91 Suppl 1:S24-33. doi: 10.1016/j. tube.2011.10.006.

10. Horna-Campos OJ, Sánchez-Pérez HJ, Sánchez I, Bedoya A, Martin M. Public transportation and pulmonary tuberculosis, Lima, Peru. Emerg Infect Dis. 2007 Oct;13(10):1491-3. doi: 10.3201/eid1310.060793.

11. Horna-Campos OJ, Bedoya-Lama A, Romero-Sandoval NC, Martin-Mateo M. Risk of tuberculosis in public transport sector workers, Lima, Peru. Int J Tuberc Lung Dis. 2010 Jun;14(6):714-9.

12. Horna-Campos OJ, Consiglio E, Sánchez-Pérez HJ, Navarro A, Cayla
JA, Martin-Mateo M. Pulmonary tuberculosis infection among workers in the informal public transport sector in Lima, Peru. Occup Environ Med. 2011 Feb;68(2):163-5. doi: 10.1136/ oem.2009.051128.

13. Powell K1, Lamb MM, Sisk MK, Federline L, Seechuk K, Lambert LA, et al. Passenger contact investigation associated with a transport driver with pulmonary tuberculosis. Public Health Rep. 2012 MarApr;127(2):202-7.

14. Gerencia de Transporte Urbano. Municipalidad Metropolitana de Lima. Resolución de Gerencia No 227 10MML/GTU. Directiva que establece la clasificación vehicular del transporte regular en Lima Metropolitana. Lima: GTU; 2010 .
15. Perú, Ministerio de Salud. Norma técnica de salud para el control de la tuberculosis. Estrategia Nacional de Prevención y Control de la Tuberculosis (ESN-PCT) [Internet]. Lima: MINSA; 2006 [citado el 16 de noviembre 2013]. Disponible en: http://www.minsa.gob.pe/pvigia/ publicaciones/tuberculosis/8Norma TB2007.pdf

Correspondencia: Octavio Garaycochea Mendoza Del Solar

Dirección: Calle Monte Carlo 212, Dpto 302, Chacarilla, Santiago de Surco, Lima, Perú

Teléfono: (+51) 954118749

Correo electrónico: garaycocheaoctavio@ gmail.com 